\title{
Stability Of Plasma Configurations During Compression
}

\section{UCRL-PROC-226046}

E. L. Ruden, J. H. Hammer

November 13, 2006

Santa Fe 2006 Megagauss Conference Los Alamos, NM, United States November 5, 2006 through November 10, 2006 
This document was prepared as an account of work sponsored by an agency of the United States Government. Neither the United States Government nor the University of California nor any of their employees, makes any warranty, express or implied, or assumes any legal liability or responsibility for the accuracy, completeness, or usefulness of any information, apparatus, product, or process disclosed, or represents that its use would not infringe privately owned rights. Reference herein to any specific commercial product, process, or service by trade name, trademark, manufacturer, or otherwise, does not necessarily constitute or imply its endorsement, recommendation, or favoring by the United States Government or the University of California. The views and opinions of authors expressed herein do not necessarily state or reflect those of the United States Government or the University of California, and shall not be used for advertising or product endorsement purposes. 


\title{
STABILITY OF PLASMA CONFIGURATIONS DURING COMPRESSION
}

\author{
Edward L. Ruden, Air Force Research Laboratory, Directed Energy Directorate, \\ Kirtland AFB, NM, USA \\ James H. Hammer, Lawrence Livermore National Laboratory, Livermore, CA, \\ $U S A$
}

\begin{abstract}
Magnetized Target Fusion (MTF) efforts are based on calculations showing that the addition of a closed magnetic field reduces the driver pressure and rise time requirements for inertial confinement fusion by reducing thermal conductivity. Instabilities that result in convective bulk transport at the Alphen time scale are of particular concern since they are much faster than the implosion time. Such instabilities may occur during compression due to, for example, an increase in the plasma-magnetic pressure ratio $\beta$ or, in the case of a rotating plasma, spin-up due to angular momentum conservation. Details depend on the magnetic field topology and compression geometry.

A hard core z pinch with purely azimuthal magnetic field can theoretically be made that relaxes into a wall supported diffuse profile satisfying the Kadomtsev criterion for the stability of $m=0$ modes, which is theoretically preserved during cylindrical outer wall compression. The center conductor radius and current must also be large enough to keep the $\beta$ below stability limits to stabilize modes with $m>0$. The stability of $m>0$ modes actually improves during compression. A disadvantage of this geometry, though, is plasma contact with the solid boundaries. In addition to the risk of high $\mathrm{Z}$ impurity contamination during the (turbulent) relaxation process, contact thereafter can cause plasma pressure near the outer surface to drop, violating the Kadomtsev criterion locally. The resultant $m=0$ instability can then convect impurities inward. Also, the center conductor (which is not part of the Kadomtsev profile) can go $m=0$ unstable, convecting impurities outward. One way to mitigate impurity convection is to instead use a Woltjer-Taylor minimum magnetic energy configuration (spheromak). The sheared magnetic field inhibits convection, and the need for the center conductor is eliminated. The plasma, however, would likely still have to be wall supported due to unfavorable $\beta$ scaling during quasispherical (3-D) compression otherwise.

Use of a Field Reversed Configuration (FRC) substantially resolves the wall contact issue, but at the cost of introducing a new (rotational) instability. An FRC has an open magnetic field outside a separatrix which effectively diverts wall material. However, FRC particles diffusing across the separatrix have a preferred angular momentum, causing the FRC within to counter-rotate in response. When the FRC's rotational-diamagnetic drift frequency ratio $\alpha$ reaches a critical value of order unity, the FRC undergoes a rotational instability that results in rapid particle loss. The instability is exacerbated by cylindrical compression since $\beta \sim$ $R^{-2 / 5}$ during this phase, assuming angular momentum conservation. A multipole magnetic field frozen into the solid liner during compression may stabilize this mode directly and/or by impeding spin-up without significantly perturbing the implosion's azimuthal symmetry.
\end{abstract}




\section{HARD CORE Z PINCH}

The adiabatic ideal MHD response of a hard core $\mathrm{z}$ pinch with purely azimuthal magnetic field and inner radius $a$ to an outer conductor of initial radius $r=b_{0}$ compressing to $r=b$ may be determined from the radius $r=r\left(r_{0}\right)$ of fluid elements initially at radii $r_{0}$ (a Lagrangian parameterization). If the compression occurs slowly enough that the plasma approximates an equilibrium at all times, this satisfies[11]

$$
\frac{d}{d r_{0}}\left[p_{0}\left(\frac{r}{r_{0}} \frac{d r}{d r_{0}}\right)^{-5 / 3}\right]+\frac{1}{2 \mu_{0} r^{2}} \frac{d}{d r_{0}}\left[\left(r B_{0}\right)^{2}\left(\frac{d r}{d r_{0}}\right)^{-2}\right]=0
$$

The "0" subscript will identify an initial profile property, except for $Q_{0}$ and $\mu_{0}$. This ordinary differential equation (ODE) may be rewritten in terms of $u=r^{2}$ vs. $r_{0}$, and solved for $u^{\prime \prime}=d^{2} u / d r_{0}^{2}$. Given an arbitrary initial equilibrium state, then, $u\left(r_{0}\right)$ for a compressed state may be evaluated numerically given boundary conditions $u(a)=a$, and some value of $u^{\prime}=d u / d r_{0}$ at $r_{0}=a$. From this, one then finds $u^{\prime \prime}(a)$ from the ODE. $u\left(a+\triangle r_{0}\right)$ and $u^{\prime}\left(r_{0}+\triangle r_{0}\right)$ may then be parabolically and linearly extrapolated, respectively, where $\Delta r_{0}$ is a small increment. Plugging these back into the ODE, one then finds $u^{\prime \prime}\left(a+\Delta r_{0}\right)$, from which one may proceed to extrapolate $u^{\prime}\left(a+2 \triangle r_{0}\right)$ and $u^{\prime \prime}\left(a+2 \triangle r_{0}\right)$. The process is repeated until one reaches $r_{0}=b_{0}$, at which time the value of $b=\sqrt{u\left(r_{0}\right)}$ of the solution is determined ex post facto.

Density $\rho$, magnetic field $B$, pressure $p$, and $\beta=2 \mu_{0} p / B^{2}$ may then be found from[11]

$$
\begin{array}{ll}
\rho=\rho_{0}\left(\frac{r}{r_{0}} \frac{d r}{d r_{0}}\right)^{-1} & B=B_{0}\left(\frac{d r}{d r_{0}}\right)^{-1} \\
p=p_{0}\left(\frac{r}{r_{0}} \frac{d r}{d r_{0}}\right)^{-5 / 3} & \beta=\beta_{0}\left(\frac{r_{0}}{r}\right)^{2}\left(\frac{r}{r_{0}} \frac{d r}{d r_{0}}\right)^{1 / 3}
\end{array}
$$

The Kadomtsev criteria[5] for stability of all azimuthal modes $\{m\}$ for the compressed state are then used to determine stability

$$
\begin{array}{ll}
Q_{0}=\frac{-(6+5 \beta)}{20} \frac{r}{p} \frac{d p}{d r}<1 & (m=0) \\
Q_{1}=-\beta \frac{r}{p} \frac{d p}{d r}<1 & (m \geq 1)
\end{array}
$$

The above solution is required in general. In the rest of this section, though, we will focus on the special case of an initial "Kadomtsev profile", which, except for the numerical evalution a two key parameters, has an analytic solution. This is of special interest because a hard core z-pinch can theoretically be made that relaxes roughly into this profile that marginally satisfies the Kadomtsev criteria by having $Q_{0}=1$ everywhere[6]. Given this profile for our initial state, we have the following properties parameterized by $\beta_{0}$,

$$
\begin{array}{cc}
\frac{p_{0}}{p_{a 0}}=\frac{\beta_{0}^{5 / 2}\left(4+5 \beta_{a 0}\right)^{5 / 2}}{\beta_{a 0}^{5 / 2}\left(4+5 \beta_{0}\right)^{5 / 2}} & \frac{r_{0}}{a}=\frac{\beta_{a 0}^{3 / 4}\left(4+5 \beta_{0}\right)^{1 / 4}}{\beta_{0}^{3 / 4}\left(4+5 \beta_{a 0}\right)^{1 / 4}} \\
\frac{B_{0}}{B_{a 0}}=\frac{\beta_{0}^{3 / 4}\left(4+5 \beta_{a 0}\right)^{5 / 4}}{\beta_{a 0}^{3 / 4}\left(4+5 \beta_{0}\right)^{5 / 4}} & Q_{1}=\frac{20 \beta_{0}\left(5 \beta_{0 a}+4\right)^{5 / 2}}{\left(5 \beta_{0}+6\right)\left(5 \beta_{0}+4\right)^{5 / 2}}
\end{array}
$$

Here and elsewhere, subscripts " $a$ " and " $b$ " identify property values at $r=a$ and $r=b$, respectively. $Q_{1}$ falls off from monotonically from it's maximum at $r_{0}=a$. The configuration is marginally stable to $m \geq 1$, with $Q_{a 1}=1$, if $\beta_{a 0}=2 / 5$. 
The time invariant mass and magnetic flux per unit axial length within a fluid element of a general ideal MHD pinch bounded by $r_{0}$ and $r_{0}+d r_{0}$ during compression are $2 \pi r \rho d r_{0}$ and $B d r_{0}$, respectively. The ratio, then, is a time invariant of the fluid element (parameterized by $r_{0}$ ) called the Kadamtsev parameter $K=K\left(r_{0}\right)$. Expressing in terms of $p$ instead of $\rho$ via the adiabatic invariant $p \rho^{-5 / 3}$, we have

$$
K\left(r_{0}\right)=\frac{p^{3 / 5} r}{B}
$$

One finds from substitution that $K\left(r_{0}\right)$ is uniform for a Kadomtsev profile so, when adiabatically compressed,

$$
\frac{p^{3 / 5} r}{B}=\frac{p_{a 0}^{3 / 5} a}{B_{a o}}
$$

is a constant of both time and space.

If we furthermore assume that the plasma remains in equilibrium, Eq. 6 and the definition of $\beta$ may be used to express the pressure balance equation

$$
\frac{d p}{d r}+\frac{1}{2 \mu_{0} r^{2}} \frac{d}{d r}(r B)^{2}=0
$$

as a differential equation in $p$ with reference to $\beta$, with the solution being a Kadomtsev profile. That is, the uniformity of $p^{3 / 5} r / B$ implies and is implied by (is equivalent to) a Kadomstev profile, and the plasma remains in one during compression. So,

$$
\begin{array}{rrrl}
\frac{p}{p_{a}} & =\frac{\beta^{5 / 2}\left(4+5 \beta_{a}\right)^{5 / 2}}{\beta_{a}^{5 / 2}(4+5 \beta)^{5 / 2}} & \frac{r}{a} & =\frac{\beta_{a}^{3 / 4}(4+5 \beta)^{1 / 4}}{\beta^{3 / 4}\left(4+5 \beta_{a}\right)^{1 / 4}} \\
\frac{B}{B_{a}} & =\frac{\beta^{3 / 4}\left(4+5 \beta_{a}\right)^{5 / 4}}{\beta_{a}^{3 / 4}(4+5 \beta)^{5 / 4}} & Q_{1} & =\frac{20 \beta\left(5 \beta_{a}+4\right)^{5 / 2}}{(5 \beta+6)(5 \beta+4)^{5 / 2}}
\end{array}
$$

$Q_{0}=1$ is preserved and $m=0$ remains marginally stable.

Solutions to $\beta_{a}, B_{a}, p_{a}, Q_{1}$, and other properties such as $\rho$ and $T$ needed to complete the description and stability analysis of the compressed state are found first by choosing a value of $\beta_{a}$ and solving for those properties in terms of it. One finds from Eq. 6 solved at $r=a$ and the definition of $\beta$, for example,

$$
\frac{B_{a}}{B_{a 0}}=\left(\frac{\beta_{a 0}}{\beta_{a}}\right)^{3} \quad \frac{p_{a}}{p_{a 0}}=\left(\frac{\beta_{a 0}}{\beta_{a}}\right)^{5}
$$

From this one sees that compression, which causes $B_{a}$ and $p_{a}$ to rise, results in $\beta_{a}<\beta_{a 0}$. Since $\beta_{a} \leq 2 / 5$ is the criterion from the stability of $m \geq 1$ for a Kadomtsev profile, a configuration initially stable to these modes is stable under compression too. All modes, then, remain stable.

As with the general problem (Eq. 1), the value of $b$ for this solution is found ex post facto. From the Kadomtsev $r$ profile,

$$
\frac{b}{a}=\frac{\beta_{a}^{3 / 4}\left(4+5 \beta_{b}\right)^{1 / 4}}{\beta_{b}^{3 / 4}\left(4+5 \beta_{a}\right)^{1 / 4}}
$$

But for this we need $\beta_{b}$ too. To this end, we derive a relationship between a given fluid element's $\beta$ and its initial value $\beta_{0}$ from the fact that flux interior to that element is preserved,

$$
\int_{\beta_{a}}^{\beta} B \frac{d r}{d \beta} d \beta=\int_{\beta_{a 0}}^{\beta_{0}} B_{0} \frac{d r_{0}}{d \beta_{0}} d \beta_{0}
$$


Plugging in the initial and compressed Kadomstev profiles (Eqs. 4 and Eqs. 8), and making use of Eq. 6 and definition of $\beta$, the result is

$$
\frac{\beta_{a 0}^{3}\left(5 \beta_{a}+4\right)}{\beta_{a}^{3}\left(5 \beta_{a 0}+4\right)}\left(\frac{\ln \left(\frac{\beta\left(5 \beta_{a}+4\right)}{\beta_{a}(5 \beta+4)}\right)+20\left(\beta-\beta_{a}\right)}{\ln \left(\frac{\beta_{0}\left(5 \beta_{a 0}+4\right)}{\beta_{a 0}\left(5 \beta_{0}+4\right)}\right)+20\left(\beta_{0}-\beta_{a 0}\right)}\right)=1
$$

Solved numerically for $\beta\left(\beta_{0}\right)$ (given $\beta_{a 0}$ and $\beta_{a}$ ), we have $\beta_{b}=\beta\left(\beta_{b 0}\right)$ needed to find $b$, where $\beta_{b 0}$ is determined by numerically solving for it from the initial Kadomstev $r$ profile evaluated at $r=b_{0}$,

$$
\frac{b_{0}}{a}=\frac{\beta_{a 0}^{3 / 4}\left(4+5 \beta_{b 0}\right)^{1 / 4}}{\beta_{b 0}^{3 / 4}\left(4+5 \beta_{a 0}\right)^{1 / 4}}
$$

Incidentally, having $\beta\left(\beta_{0}\right)$ also allows one to calculate $\rho\left(\beta_{0}\right)$ and $T\left(\beta_{0}\right)$ from its initial profile via the adiabatic invariant,

$$
\frac{\rho\left(\beta_{0}\right)}{\rho_{0}\left(\beta_{0}\right)}=\left(\frac{p\left(\beta\left(\beta_{0}\right)\right)}{p_{0}\left(\beta_{0}\right)}\right)^{3 / 5} \quad \frac{T\left(\beta_{0}\right)}{T_{0}\left(\beta_{0}\right)}=\left(\frac{p\left(\beta\left(\beta_{0}\right)\right)}{p_{0}\left(\beta_{0}\right)}\right)^{2 / 5}
$$

Listed below are the results of a marginally stable Kadomtsev profile $\left(\beta_{a 0}=2 / 5\right)$ initially with $b_{0} / a=10$ compressed to the point where $\beta_{a}=0.17$, subsequently determined to correspond to $b / a=1.2$. This example was chosen to match the parameters of a published MHD simulation intended to represent this case.[11]

$$
\begin{array}{ccc}
\frac{b_{0}}{a}=10 & \beta_{a 0}=0.4 & \beta_{a}=0.17 \\
\frac{B_{a}}{B_{a 0}}=13.03 & \frac{B_{b}}{B_{b 0}}=76.77 & Q_{a 1}=0.496 \\
\frac{p_{a}}{p_{a 0}}=72.12 & \frac{p_{b}}{p_{b 0}}=4.733 \times 10^{4} & \frac{b}{a}=1.203 \\
\frac{\rho_{a}}{\rho_{a 0}}=13.03 & \frac{\rho_{b}}{\rho_{b 0}}=638.4 & \beta_{b 0}=1.633 \times 10^{-2} \\
\frac{T_{a}}{T_{a o}}=5.536 & \frac{T_{b}}{T_{b 0}}=74.14 & \beta_{b}=0.1311
\end{array}
$$

The results are consistent with the reference's Fig. 5. In addition the general properties already discussed, one noteworthy feature is the much greater compression and heating at larger radii.

In MHD simulations which include thermal conductivity, contact with the liner causes plasma pressure near the outer surface to drop, violating the Kadomtsev criterion locally. The resultant localized $m=0$ instability can then convect impurities inward, at least a small distance. Also, the center conductor (which is not part of the Kadomtsev profile) can go $m=0$ unstable, convecting impurities outward[6]. One question then is the radial extent of transport from the unstable walls, as suggested in Fig. 1. The effect of impurities can be significantly mitigated significantly if the walls are coated with $\mathrm{Li}$ or, better yet, frozen $\mathrm{D}_{2}$. 


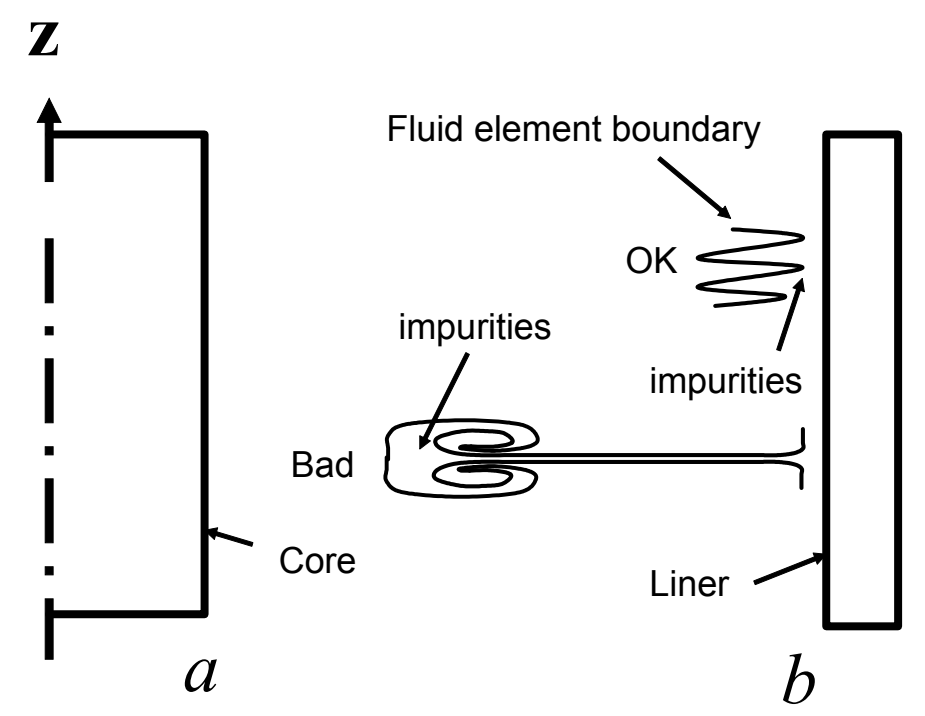

Figure 1. Hypothetical consequences of Kadomtsev unstable layer near wall.

A Woltjer-Taylor minimum magnetic energy configuration (spheromak) is an option that can mitigate such transport. The sheared magnetic field inhibits convection, and the need for the center conductor is eliminated. Such a configuration, however, is only stable for low $\beta$ if plasma pressure at the wall is to be kept small. To make matters worse, $\beta \sim R^{-1}$ during quasispherical (3-D) flux conserving selfsimilar compression, where $R$ is the liner radius. The plasma, therefore, would likely still have to be wall supported.

\section{FIELD REVERSED CONFIGURATION}

Use of a an FRC substantially resolves the wall contact issue, but at the cost of introducing a new (rotational) instability. An FRC has an open magnetic field outside a separatrix which effectively diverts wall material. However, FRC particles diffusing across the separatrix have a preferred angular momentum, causing the FRC within to counter-rotate in response.[1] When the FRC's rotational-diamagnetic drift frequency ratio $\alpha$ reaches a critical value of order unity, the FRC undergoes a rotational instability that results in rapid particle loss. This instability can be understood conceptually in terms of a simplified FLR treatment. Roberts and Taylor[8] show that for the planar R-T case where density $\rho_{0}$ increases with height as $\exp (\lambda x)$ in a gravitational field of acceleration $g$, R-T modes are stable for magnetically transverse wave numbers $k$ provided

$$
g \leq \nu^{2} \lambda k^{2} \quad \nu=\frac{k_{B} T_{i}}{2 Z e B}
$$

Here, $\nu$ is the "gyroviscosity" coefficient, with $k_{B}, Z, e$, and $B$ being the Boltzmann constant, mean ionization level, elementary charge, and magnetic field magnitude, respectively. 
To apply this result to a rotating cylindrical plasma column with an axial magnetic field of characteristic magnitude $B$, note that the ion diamagnetic drift frequency is

$$
\Omega_{D i}=-\frac{v_{D i}}{r} \quad \mathbf{v}_{D i}=-\frac{\nabla p_{i} \times \mathbf{B}}{e Z n_{i} B^{2}}
$$

where $\mathbf{v}_{D i}$ is the diamagnetic drift velocity, and $p_{i}$ and $n_{i}$ are the ion pressure and number density, respectively. Using centripetal acceleration for $g$ at the characteristic radius of the column $R_{1}$ (magnetic null radius), and wrapping the mode "plane" around the circumference, we have, then, the characteristic values,

$$
\Omega_{D i}=\frac{2 \lambda \nu}{R_{1}} \quad g=\Omega_{R}^{2} R_{1} \quad k=\frac{n}{R_{1}}
$$

Our stability criterion $g \leq \nu^{2} \lambda k^{2}$ is, then,

$$
\alpha=\frac{\Omega_{R}}{\Omega_{D i}} \leq \frac{n}{2 \sqrt{\lambda R_{1}}}
$$

Given a characteristic density gradient scale length of $1 / \lambda \approx R_{1}$, stability requires $\alpha \lesssim 1$ for the least stable mode $n=2$. Technically, $n=1$ goes unstable first, but the (planar) model applied to cylindrical geometry does not conserve lateral linear momentum for this mode, so is inapplicable. The threshold is respectably close to the threshold value observed[4] and described by more sophisticated modeling[2][9][3], given the geometrical liberties taken.

The dynamics of the $n=2$ mode is of particular concern for the MTF application because $\alpha$ theoretically increases significantly during wall compression by a conducting cylindrical liner. To show this, firstly, $\Omega_{R}$ increases in proportion to $R_{s}^{-2}$ from angular momentum conservation where $R_{s}$ is the separatrix radius. Meanwhile, $x_{s} \equiv R_{s} / R_{c}$ is conserved during cylindrical wall compression, where $R_{c}$ is the liner inner radius (Tuszewski, p. 2058).[12] Given this, plasma $\beta$ is conserved (Tuszewski, Eq. 10).[12] Given this and flux conservation, $\Omega_{D i}$ is proportional to $T_{i}$, (Shimamura, Eq. 7 with $\Omega^{*}=-\Omega_{D i}$ ).[10] The FRC's characteristic volume $V=\pi R_{s}^{2} l_{s}$, meanwhile, decreases as $R_{s}^{N}$, where $l_{s}$ is the separatrix length, and $N$ is the dimensionality of compression. Assuming adiabatic compression, $T_{i} V^{(5 / 3)-1}$ is conserved. Therefore, $\Omega_{D i} r_{s}^{2 N / 3}=$ constant, and $\Omega_{D i}$ increases in proportion to $R_{s}^{-2 N / 3} . \alpha$, then, increases in proportion to $R_{s}^{-2} / R_{s}^{-2 N / 3}=R_{s}^{-2(1-N / 3)} . N=12 / 5$ for cylindrical wall compression (Tuszewski, Table V)[12], so $\alpha$ increases in proportion to $R_{s}^{-2 / 5}$ or, equivalently, $R_{c}^{-2 / 5}$. The (target) factor of 10 radial compression, then, increases $\alpha$ by a factor of $10^{2 / 5} \approx 2.5$.

A multipole magnetic field frozen into the solid liner during compression may stabilize this mode directly and/or by impeding spin-up without significantly perturbing the implosion's azimuthal symmetry.[7]

\section{SUMMARY}

The effect on compression on the most problematic instabilities of the two leading candidates for MTF are discussed. For a hard-core pinch, radial convection of wall material is expected from the destabilization of an initial Kadomtsev profile, though further investigation is needed to assess its significance. Mitigation 
strategies such as using an overstabilized initial state and/or low Z wall loading are possible. For an FRC, disintegration during compression is expected due to unfavorable scaling of rotation $n=2$ instability's stabilitiy threshold parameter $\alpha$. Use of multipole stablization of the $n=2$ instability is an option that needs further investigation.

\section{References}

[1] E. V. Belova, R. C. Davidson, H. Ji, M. Yamada, C. D. Cothran, M. R. Brown, and M. J. Schaffer, Numerical study of the formation, ion spin-up and nonlinear stability properties of field-reversed configurations, Nucl. Fusion, 46 (2006), pp. $162-170$.

[2] J. P. Freidberg and L. D. Pearlstein, Rotational instabilities in a theta pinch, Phys. Fluids, 21 (1978), pp. 1207-1217.

[3] D. S. Harned, Rotational instabilities in the field-reversed configuration: results of hybrid simulations, Phys. Fluids, 26 (1983), pp. 1320-1326.

[4] Y. Ito, M. Tanjyo, S. Ohi, G. S., and T. Ishimura, Ion rotational velocity of a field-reversed configuration plasma measured by neutral beam probe spectroscopy, Phys. Fluids, 30 (1987), pp. 168-174.

[5] B. B. Kadomtsev, Hydromagnetic stability of a plasma, in Reviews of Plasma Physics, M. A. Leontovichm, ed., vol. 2, New York, 1966, Consultants Bureau, pp. 153-199.

[6] V. Makhin, R. E. Siemon, B. S. Bauer, A. Esaulov, I. R. Lindemuth, V. I. Sotnikov, D. D. Ryutov, and P. T. Sheehey, Self-organization observed in numerical simulations of a hard-core diffuse $Z$ pinch, Phys. Plasmas, 12 (2005), p. 042312 .

[7] D. J. Rej, W. T. Armstrong, G. A. Barnes, R. E. Chrien, W. N. Hugrass, P. L. Klingner, K. F. KcKenna, R. E. Siemon, Tuszewski, and D. S. Harned, Highpower magnetic-compression heating of field-reversed configurations, Phys. Fluids, 29 (1986), pp. 2648-2656.

[8] K. V. Roberts and J. B. Taylor, Magnetohydrodynamic equations for finite larmor, Phys. Rev. Lett., 8 (1962), pp. 197-198.

[9] C. E. Seyler, Vlasov-fluid stability of a rotating theta pinch, Phys. Fluids, 22 (1978), pp. 2324-2330.

[10] S. Shimamura and Y. Nogi, Helical quadrupole field stabilization of fieldreversed configuration plasma, Fusion Tech., 9 (1978), pp. 69-74.

[11] R. E. Siemon, W. Atchison, T. Awe, B. Bauer, A. Buyko, V. Chernyshev, T. Cowan, J. Degnan, S. Faehl, R.J. Fuelling, S. Garanin, T. Goodrich, A. Ivanovsky, I. Lindemuth, V. Makhin, V. Mokhov, R. Reinovsky, D. Ryutov, D. Scudder, T. Taylor, and V. Yakubov, Stability analysis and numerical simulation of a hard-core diffuse $z$ pinch during compression with atlas facility liner parameters, Nucl. Fusion, 45 (2005), pp. 1148-1155. 
[12] M. Tuszewski, field reversed configurations, Nuclear Fusion, 28 (1988), pp. 2033-2092.

This work was performed under the auspices of the U.S. Department of Energy by the University of California, Lawrence Livermore National Laboratory under Contract No. W-7405-Eng-48. 